\title{
Estimates of heterosis and combining ability of soybean diallel crossings
}

\author{
Jose Ricardo Bagateli ${ }^{1^{*}}$, Carlos André Bahry ${ }^{2}$, Raimunda Nonata Oliveira da Silva ${ }^{1}$, Ivan Ricardo Carvalho ${ }^{1}$, \\ Giordano Gelain Conte ${ }^{1}$, Francisco Amaral Villela ${ }^{1}$, Gizele Ingrid Gadotti ${ }^{1}$, Geri Eduardo Meneghello ${ }^{1}$
}

\author{
${ }^{1}$ College of Agronomy Eliseu Maciel, Federal University of Pelotas, Pelotas, RS, Brazil \\ ${ }^{2}$ Federal Technological University of Paraná, Dois vizinhos, PR, Brazil
}

\author{
*Corresponding author: ricardobagateli@gmail.com
}

\section{Abstract}

This study aimed to estimate the general and specific combining ability of partial soybean diallel crossings. The parents were divided in two groups contrasting for the characteristics of mass of thousand seeds, maturity group, flowers' color, lodging and growth aspect. The scheme of partial diallel followed the Griffing model, where it were included the parents and the $F_{1}$ generation. There was predominance of additive genic effects for the characteristics number of seeds per pods, number of seeds per plant and plants height. For number of pods per plant, seeds per plant and mass of hundred seeds the dominance effects were expressed by the superiority of specific combining ability in the determination of these characters. The parents G3 and G7 were the ones that contributed the most to the increase in yield in function of general combining ability for the characters number of pods per plant, seeds per plant, plant yield and mass of hundred seeds. The hybrids from the crossings between G3 x G5 and G3 x G4 are more promising for the characteristics related to yield, since they present elevated heterosis effect and high specific combining ability associated to increase general combining ability presented by the parent $\mathrm{G} 3$.

Keywords: Glycine max L., plant breeding, additive and no-additive genetics effects, quantitative genetic, genes and alleles combination.

Abbreviations: GCA- general combining ability; MSP- mass of seeds per plant; M100- mass of hundred seeds; NPP- number of pods per plant; NSP- number of seeds per pod; NSPP- number of seeds per plant; PH- plant height in R8; SCA- specific combining ability.

\section{Introduction}

The soybean is grown in a range of environments and its diffusion into all Brazilian regions occurred, mainly, due to genetic breeding. Cultivars with wide adaptation, stability and exalted productive potential are being incorporated to the market, what propitiates increases in the grains yield (Polizel et al., 2013).

In the soybean breeding, the choice of parents to breed is, generally, a hard task to be executed, due to the existence of a large number of genotypes with elevated potential for the attributes of interest (Borém and Miranda, 2009). In order to minimize these effects, breeders take base on important information of these parents, such as, agronomic performance, genetic distance, combining ability and its behavior per se (Carvalho et al., 2017).

The selection of parents based on its phenotype might not be enough to predict the performance of the recombined progenies, since it is sought allelic and genic complementarity in order to maximize the combining ability, benefiting of its additive genetic effects (Lorencetti et al., 2005). When identifying the potential parents, these are artificially crossed aiming to obtain transgressive heterozygous combinations and maximize the segregating populations variability, thus, proportionating genetic gains to the character in evidence, as well, incrementing the probability to select superior segregating families (Carvalho et al., 2018; Carvalho et al., 2016).

These limitations could be avoided through the choice of promising parents, identifying in the early generations its ability in directing alleles and genes of interest (Ramalho et al., 2012). These strategies can reduce, in the early generations, some issues with incompatibility, low complementarity and combining ability (Borém and Miranda, 2009). Efficient biometric approaches may identify prospective parents, which could compose the crossing blocks and, consequently, boost the obtaining of transgressive recombination (Valério et al., 2009). However, there is a lack of information about the soybean breeding using early generations, most due to the difficulty to proceed crosses and $F_{1}$ heterozygotes (Daronch et al., 2014). Aiming to minimize these adversities, some strategies with diallel crosses might be used, seeking to estimate the general combining ability (GCA), which represents the sum of additive genic effects, while the specific combining ability (SCA) base on the non-additive genetic deviances (Cruz et al., 2011; Mebrahtu and Devine, 2009).

The combining ability assist the breeder in the identification of superior genotypes that reveals the agronomic ideotype necessary to attend the breeding program requirements (Nassar, 2013). In this sense, the GCA refers to the average 
performance of the determinant genes to that specific character, while the SCA explores the dominance, overdominance and epistatic deviances (Daronch et al., 2014). In this context, it was aimed to estimate the general and specific combining ability of partial soybean diallel crossings.

\section{Results}

\section{Means, genotypic correlation and diallelic analysis}

The estimates of genotypic correlation among the studied variables (Table 2) revealed that NPP is highly correlated to NSPP, and the last one, was the most responsible for MSP, indicating that these characteristics are the most promising in assisting the selection of the most productive plants. It is pointed out that these characters presented low correlation with the mass of hundred seeds (M100).

In soybeans, Morceli Junior et al. (2008), verified that NPP, NSP, NSPP and M100 have a high and positive correlation, indicating that exists inter-relations between these characters, what allows targeting the selection of the most productive progenies, mostly, through higher NSPP.

The variance analysis of the characteristics NPP, NSPP, NSP, MSP, M100 and PH indicate existence of variability between eight parents, observed by the significance of the treatment effects $(p<0,01)$, according Table 3 .

When comparing the groups means (Table 4), it was verified that the parents of the group $1\left(G_{1}\right)$ presented higher PP, besides a lower NSPP. It is possible because these parents presented $M 10026.8 \%$ superior to the group $2\left(G_{2}\right)$. The parents of $G_{2}$ had, in general, higher $\mathrm{PH}$. Despite these groups presenting higher NPP and NSPP, its MSP was $2.6 \%$ lower than $G_{1}$. These results were expected, since the parents of $G_{1}$ were selected for showing more favorable phenotypes to $M 100$ than the ones in $G_{2}$.

When contrasting the mean of hybrids with the parents, it is observed that the hybrids were more productive than the parents of both groups, reaching $6.3 \%$ and $8 \%$ for MSP and NSPP, respectively. For the characteristics NSP and M100, the hybrids mean was inferior to the parents' mean, and for $\mathrm{PH}$, the hybrids means was superior to the parents' mean.

The parent of $G_{1}$, the $G 3$ genotype, was the one that originated hybrids with higher yield. The combinations $\mathrm{G} 3 \mathrm{x}$ G6, G3 $\times$ G4 and G3 $\times$ G7 were the most productive, with $27.1 ; 23.6$ and 22.6 grams per plant ${ }^{-1}$, respectively. For M100, the best hybrids were the combination of $\mathrm{G} 1 \times \mathrm{G} 4$ with $14.1 \mathrm{~g}$, followed by G3 x G7 with $14 \mathrm{~g}$. According with Silva (2011), these results indicate the existence of heterosis for MSP, NSPP and PH, results of positive dominance deviances for the genes determinant of MSP and negative for NSP and M100.

The Table 5 shows the summary of diallelic analysis, in which the source of variation "treatments" was deployed in effects of general and specific combining ability and in the contrast between means of the two groups of parents $\left(G_{1} v s G_{2}\right)$.

It was identified significant difference $(p<0.01)$ between the groups' means for all variables, with exception of MSP. For GCA and SCA all characteristics differ statistically $(p<0.01)$. The significance of the contrast between the groups' means $\left(G_{1}\right.$ vs $\left.G_{2}\right)$ for most of the analyzed variables indicates genetic diversions within the two genetic groups, being it such an aid to the possibility of genetic gains in the development of future breeding works with its hybrid populations. The genotypes of the $G_{1}$ stands out by its higher M100 and MSP. In the other hand, the $G_{2}$ genotypes stands out by its higher NPP and NSPP.

The results evidence higher importance of the GCA in relation to the SCA for NSP, MSP and $\mathrm{PH}$, with the predominance of additive genetic effects, expressed by the superiority of the sum of squares of the GCA. The opposite can be observed for NPP, NSPP and M100, with higher contribution of the dominance effects in the determination of these characters. In this case, it was observed superiority of the sum of squares of SCA in relation to the sum of squares of GCA.

\section{Discussion}

It is worth to point out the estimates of GCA depends on the genetic difference of the parents and the average effect of the allelic replacement in the other group and are associated to the additive effects. The SCA, for instance, is function of the dominance effect and the product of the difference of allelic frequencies of the opposite groups parents, making that the same stay related to the dominance and epistatic effects (Cruz, Regazzi and Carneiro 2012).

It is important to highlight that there only a few studies with diallelic analysis related to yield components of soybean seeds. The significances registered indicates existence of variability, that results in the action of additive and nonadditive genic effects, and indicates the possibility of obtaining promising lines for a soybean breeding program. Similar results were also found by Zorzerto et al. (2008). In other species, the GCA was superior to SCA for the yield components, as stated by Baldissera et al. (2012) in common beans.

\section{Estimates of General (GCA) and Specific Combining Ability}

The estimates of the $\operatorname{GCA}\left(\hat{g}_{i}\right)$ effects for all evaluated characters are showed in the Table 6 . Cruz, Regazzi and Carneiro (2012) states that, when found low estimates of $\hat{g}_{i}$, positive or negative, the value of GCA of the parent calculated based on its crossings with the other parents, it does not differs largely from the general mean of the diallelic crossings. When high estimates of $\hat{g}_{i}$ are found, besides being positive or negative, the parent in question is much more superior or inferior than the others in the diallel, in relation to the average behavior of the crossings.

It is verified that for the characteristics NPP, NSPP, MSP and M100, only the parents G3 and G7 accused positive values of $\hat{g}_{i}$ for all, simultaneously. It allows us to affirm that in the crossings, in which these parents participate, there will be contribution for the increase in yield. In the other hand, the parents G4 and G8 revealed negative values of $\hat{g}_{i}$ for NPP, NSPP, MSP and M100. Thus, these parents must not be recommended for breeding programs. It is worth to emphasize that the parent $G 6$, from $G_{2}$, also stood out in relation to the estimates of $\hat{g}_{i}$, being these highly positives 
Table 1. Partial diallel scheme, being eight Parents and fifteen $F_{1}$ hybrids.

\begin{tabular}{|c|c|c|c|c|c|c|}
\hline \multirow{2}{*}{$\begin{array}{l}\text { Parents } \\
\text { Paternal parent }\end{array}$} & & \multicolumn{5}{|c|}{$\begin{array}{c}\text { Parent Maternal } \\
\text { Group } 2 \\
\end{array}$} \\
\hline & & G4 & G5 & G6 & G7 & G8 \\
\hline Group 1 & $\mathrm{n}^{\circ}$ & 4 & 5 & 6 & 7 & 8 \\
\hline G1 & 1 & $1 \times 4$ & $1 \times 5$ & $1 \times 6$ & $1 \times 7$ & $1 \times 8$ \\
\hline G2 & 2 & $2 \times 4$ & $2 \times 5$ & $2 \times 6$ & $2 \times 7$ & $2 \times 8$ \\
\hline G3 & 3 & $3 \times 4$ & $3 \times 5$ & $3 \times 6$ & $3 \times 7$ & $3 \times 8$ \\
\hline
\end{tabular}

Table 2. Genotypic correlations estimates between the studied variables in the diallelic analysis between eight soybean parents, in partial diallel scheme.

\begin{tabular}{|c|c|c|c|c|c|c|}
\hline & NPP & NSPP & NSP & MSP (g) & M100 (g) & $\mathrm{PH}(\mathrm{cm})$ \\
\hline NPP & - & $0.714^{++}$ & $-0.7147^{++}$ & $0.5546^{++}$ & $-0.3700^{++}$ & $0.5663^{++}$ \\
\hline NSPP & & - & $-0.0539^{++}$ & $0.8095^{++}$ & $-0.4388^{++}$ & $0.5692^{++}$ \\
\hline NSP & & & - & $-0.0060^{++}$ & $0.1072^{++}$ & $-0.3206^{++}$ \\
\hline MSP (g) & & & & - & $0.1659^{++}$ & $0.3007^{++}$ \\
\hline M100 (g) & & & & & - & $-0.4864^{++}$ \\
\hline $\mathrm{PH}(\mathrm{cm})$ & & & & & & - \\
\hline
\end{tabular}

NPP - Number of pods per plant, NSPP - Number of seeds per plant, NSP - Number of seeds per pod, MSP - Mass of seeds per plant, M100 - Mass of hundred seeds, PH - Plant height, ${ }^{++}$- Significant at $5 \%$ by the bootstrap method with 5.000 simulations.

Table 3. Summary of variance analysis between eight soybean parents, in a partial diallel without inclusion of reciprocals.

\begin{tabular}{|c|c|c|c|c|c|c|c|}
\hline \multirow{2}{*}{ SV } & \multirow{2}{*}{ DF } & \multicolumn{6}{|c|}{ Mean Square } \\
\hline & & NPP & NSPP & NSP & MSP (g) & M100 (g) & $\mathrm{PH}(\mathrm{cm})$ \\
\hline Block & 2 & 32.19 & 116.36 & 0.001 & 13.10 & 0.06 & 6.78 \\
\hline Treatment & 22 & $671.11^{* *}$ & $1268.49^{* *}$ & $0.21^{* *}$ & $19.00^{* *}$ & $4.03^{* *}$ & $137.32^{* *}$ \\
\hline Residue & 44 & 70.69 & 178.97 & 0.01 & 2.49 & 0.11 & 3.70 \\
\hline CV (\%) & & 10.25 & 8.94 & 1.86 & 7.98 & 13.29 & 1.88 \\
\hline Mean & & 82.01 & 149.71 & 5.4 & 19.78 & 2.5 & 102.86 \\
\hline
\end{tabular}

SV-Source of variation, DF-Degrees of freedom, NPP - Number of pods per plant, NSPP - Number of seeds per plant, NSP - Number of seeds per pod, MSP - Mass of seeds per plant, M100 - Mass of hundred seeds, PH - Plant height, CV - Coefficient of variation," - Significant at $p>0,01$ by F test.

Table 4. Means obtained in the evaluation of eight parents and 15 soybean hybrids, in a partial diallel, without inclusion of reciprocals, between the groups 1 and 2 .

\begin{tabular}{|c|c|c|c|c|c|c|}
\hline Parents & NPP & NSPP & NSP & MSP (g) & M100 (g) & $\mathrm{PH}(\mathrm{cm})$ \\
\hline \multicolumn{7}{|l|}{ Group $1\left(\mathrm{G}_{1}\right)$} \\
\hline G1 & 59,3 & 108,0 & 1,8 & 17,9 & 16,6 & 93,0 \\
\hline $\mathrm{G} 2$ & 49,3 & 129,0 & 2,6 & 18,6 & 14,5 & 90,0 \\
\hline G3 & 65,0 & 135,3 & 2,1 & 21,5 & 15,9 & 95,3 \\
\hline $\mathrm{G}_{1}$ Mean & 57,9 & 124,1 & 2,2 & 19,3 & 15,6 & 92,8 \\
\hline \multicolumn{7}{|l|}{ Group $2\left(\mathrm{G}_{2}\right)$} \\
\hline G4 & 78,3 & 156,3 & 2,0 & 17,4 & 11,2 & 111,7 \\
\hline G5 & 88,7 & 145,0 & 1,6 & 18,4 & 12,7 & 116,3 \\
\hline G6 & 102,7 & 179,0 & 1,7 & 21,7 & 12,1 & 105,0 \\
\hline G7 & 99,0 & 157,0 & 1,6 & 20,4 & 13,0 & 104,7 \\
\hline G8 & 75,0 & 128,7 & 1,7 & 16,1 & 12,6 & 100,7 \\
\hline $\mathrm{G}_{2}$ Mean & 88,7 & 153,2 & 1,7 & 18,8 & 12,3 & 107,7 \\
\hline Parents Mean & 77,2 & 142,3 & 1,9 & 19,0 & 13,6 & 102,1 \\
\hline Crossings & NPP & NSPP & NSP & MSP (g) & M100 (g) & $\mathrm{PH}(\mathrm{cm})$ \\
\hline $\mathrm{G} 1 \times \mathrm{G} 4$ & 86,7 & 149,3 & 1,7 & 19,8 & 13,2 & 99,7 \\
\hline $\mathrm{G} 1 \times \mathrm{G} 5$ & 78,7 & 137,0 & 1,7 & 19,3 & 14,1 & 110,0 \\
\hline $\mathrm{G} 1 \times \mathrm{G} 6$ & 105,3 & 149,3 & 1,4 & 19,9 & 13,3 & 98,0 \\
\hline G1x G7 & 92,3 & 155,3 & 1,7 & 20,7 & 13,3 & 101,3 \\
\hline $\mathrm{G} 1 \times \mathrm{G} 8$ & 73,7 & 127,7 & 1,7 & 16,6 & 13,0 & 96,3 \\
\hline $\mathrm{G} 2 \times \mathrm{G} 4$ & 58,7 & 144,0 & 2,5 & 17,8 & 12,4 & 100,7 \\
\hline $\mathrm{G} 2 \times \mathrm{G} 5$ & 84,7 & 167,3 & 2,0 & 21,0 & 12,5 & 107,3 \\
\hline $\mathrm{G} 2 \times \mathrm{G} 6$ & 81,3 & 153,7 & 1,9 & 19,4 & 12,7 & 105,0 \\
\hline G2x G7 & 76,0 & 140,0 & 1,9 & 18,7 & 13,4 & 110,3 \\
\hline $\mathrm{G} 2 \times \mathrm{G} 8$ & 74,7 & 131,7 & 1,8 & 16,3 & 12,4 & 96,7 \\
\hline $\mathrm{G} 3 \times \mathrm{G} 4$ & 95,3 & 181,0 & 1,9 & 23,6 & 13,0 & 109,3 \\
\hline $\mathrm{G} 3 \times \mathrm{G} 5$ & 82,3 & 153,7 & 1,9 & 20,5 & 13,4 & 103,7 \\
\hline $\mathrm{G} 3 \times \mathrm{G} 6$ & 100,3 & 203,7 & 2,0 & 27,1 & 13,3 & 106,3 \\
\hline G3x G7 & 99,7 & 161,7 & 1,6 & 22,6 & 14,0 & 109,7 \\
\hline G3x G8 & 79,3 & 149,7 & 1,9 & 20,0 & 13,3 & 95,0 \\
\hline Hybrids Mean & 84,6 & 153,7 & 1,8 & 20,2 & 13,2 & 103,3 \\
\hline
\end{tabular}


Table 5. Summary of diallelic analysis of the groups 1 and 2, and its hybrid combinations, in a partial diallel between eight soybean parents.

\begin{tabular}{|c|c|c|c|c|c|c|c|c|c|c|c|c|}
\hline & \multicolumn{12}{|c|}{ Variation source } \\
\hline & \multicolumn{2}{|c|}{ Treatments } & \multicolumn{2}{|l|}{$\mathrm{GCA}_{1}$} & \multicolumn{2}{|l|}{$\mathrm{GCA}_{2}$} & \multicolumn{2}{|l|}{ SCA } & \multicolumn{2}{|l|}{$\mathrm{G}_{1} v s \mathrm{G}_{2}$} & \multicolumn{2}{|l|}{ Residue } \\
\hline & SQ & MS & SQ & MS & SQ & MS & SQ & MS & SQ & MS & SQ & MS \\
\hline NPP & 14764.3 & $671.1^{* *}$ & 2265.2 & $1132.6^{* *}$ & 3869.2 & $967.3^{* *}$ & 4325.0 & $288.3^{* *}$ & 14304.7 & $4304.7^{* *}$ & 443110.3 & 70.6 \\
\hline NSPP & 27906.8 & $1268.4^{* *}$ & 5884.9 & $2992.4^{* *}$ & 8763.7 & $2190.9^{* *}$ & 9753.2 & $650.2^{* *}$ & 13404.9 & $3404.9^{* *}$ & 447874.6 & 178.9 \\
\hline NSP & 4.7 & $0.2^{* *}$ & 1.7 & $1.0^{* *}$ & 0.6 & $0.9^{* *}$ & 1.2 & $0.2^{* *}$ & 0.9 & $0.9^{* *}$ & 0.4 & 0.1 \\
\hline MSP & 418.0 & $19.0^{* *}$ & 145.0 & $72.5^{* *}$ & 142.3 & $35.6^{* *}$ & 126.0 & $8.4^{* *}$ & 14.6 & $4.6^{\mathrm{ns}}$ & 44019.9 & 24.9 \\
\hline M100 & 88.8 & $4.0^{* *}$ & 12.2 & $6.1^{* *}$ & 7.5 & $1.8^{* *}$ & 14.1 & $0.9^{* *}$ & 154.8 & $54.8^{* *}$ & 444.8 & 0.1 \\
\hline $\mathrm{PH}$ & 3021.1 & $137.3^{* *}$ & 92.7 & $46.3^{* *}$ & 952.2 & $238.0^{* *}$ & 847.5 & $56.5^{* *}$ & 11128.6 & $1128.6^{* *}$ & 44163.1 & 3.7 \\
\hline DF & 22 & & 2 & & 4 & & 15 & & 1 & & 44 & \\
\hline
\end{tabular}

Table 6. Estimates of general combining ability effects $\left(\hat{g}_{i}\right)$ between soybean parents, relative to the groups 1 and 2.

\begin{tabular}{|c|c|c|c|c|c|c|}
\hline \multirow{2}{*}{ Parents (Group 1) } & \multicolumn{6}{|l|}{$\mathrm{GCA}_{1}$} \\
\hline & NPP & NSPP & NSP & $\mathrm{MSP}(\mathrm{g})$ & M100 (g) & $\mathrm{PH}(\mathrm{cm})$ \\
\hline G1 & 1.84 & -9.09 & -0.18 & -0.86 & 0.34 & -1.18 \\
\hline $\mathrm{G} 2$ & -7.20 & -2.43 & 0.18 & -10.24 & -0.54 & -0.22 \\
\hline G3 & 5.36 & 11.53 & -0.002 & 18.90 & 0.19 & 1.41 \\
\hline \multirow{2}{*}{ Parents (Group 2) } & \multicolumn{6}{|l|}{$\mathrm{GCA}_{2}$} \\
\hline & NPP & NSPP & NSP & MSP (g) & M100 (g) & $\mathrm{PH}(\mathrm{cm})$ \\
\hline G4 & -4.84 & 2.80 & 0.15 & -0.33 & -0.44 & 1.11 \\
\hline G5 & -1.20 & -2.77 & -0.15 & -0.88 & 0.18 & 4.06 \\
\hline G6 & 8.72 & 13.89 & -0.24 & 1.65 & -0.74 & -0.83 \\
\hline G7 & 4.96 & 0.51 & -0.09 & 0.64 & 0.35 & 0.78 \\
\hline G8 & -7.65 & -14.43 & -0.02 & -1.87 & -0.02 & -5.12 \\
\hline
\end{tabular}

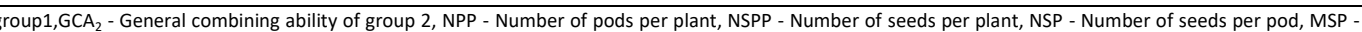
Mass of seeds per plant, M100-Mass of hundred seeds, PH - Plant height.

Table 7. Estimates of specific combining ability $\left(\hat{s}_{i j}\right)$ effects between the parents of groups 1 and 2.

\begin{tabular}{|c|c|c|c|c|c|c|c|}
\hline Crossing $\left(\mathrm{G}_{1}\right.$ vs $\left.\mathrm{G}_{2}\right)$ & NPP & & NSPP & NSP & $\operatorname{MSP}(\mathrm{g})$ & M100 (g) & $\mathrm{PH}(\mathrm{cm})$ \\
\hline $\mathrm{G} 1 \times \mathrm{G} 4$ & & 8.83 & 6.96 & -0.15 & 1.13 & -0.11 & -2.52 \\
\hline $\mathrm{G} 1 \times \mathrm{G} 5$ & & -2.82 & 0.21 & 0.04 & 0.39 & 0.13 & 4.85 \\
\hline $\mathrm{G} 1 \times \mathrm{G} 6$ & & 13.93 & -4.12 & -0.02 & -0.75 & -0.41 & -2.24 \\
\hline G1x G7 & & 4.69 & 15.25 & 0.08 & 1.06 & -0.79 & -0.52 \\
\hline $\mathrm{G} 1 \times \mathrm{G} 8$ & & -1.35 & 2.54 & 0.06 & -0.48 & -0.71 & 0.37 \\
\hline $\mathrm{G} 2 \times \mathrm{G} 4$ & & -10.12 & -5.03 & 0.24 & -0.67 & -0.07 & -2.49 \\
\hline $\mathrm{G} 2 \times \mathrm{G} 5$ & & 12.21 & 23.87 & -0.05 & 2.25 & -0.53 & 1.22 \\
\hline $\mathrm{G} 2 \times \mathrm{G} 6$ & & -1.03 & -6.45 & -0.14 & -1.02 & -0.14 & 3.79 \\
\hline $\mathrm{G} 2 \times \mathrm{G} 7$ & & -2.61 & -6.74 & -0.14 & -0.74 & 0.12 & 7.51 \\
\hline $\mathrm{G} 2 \times \mathrm{G} 8$ & & 8.68 & -0.12 & -0.28 & -0.62 & -0.49 & -0.25 \\
\hline $\mathrm{G} 3 \times \mathrm{G} 4$ & & 13.99 & 18.02 & -0.13 & 2.17 & -0.11 & 4.54 \\
\hline $\mathrm{G} 3 \times \mathrm{G} 5$ & & -2.68 & -3.75 & 0.03 & -1.09 & -0.44 & -4.07 \\
\hline $\mathrm{G} 3 \times \mathrm{G} 6$ & & 5.41 & 29.57 & 0.17 & 3.76 & -0.21 & 3.49 \\
\hline G3x G7 & & 8.52 & 0.95 & 0.15 & 0.28 & -0.04 & 5.21 \\
\hline G3x G8 & & 0.79 & 3.91 & 0.03 & 0.12 & -0.22 & -3.55 \\
\hline
\end{tabular}

$\mathrm{G}_{1}$ - Group 1, $\mathrm{G}_{2}$ - Group 2, NPP - Number of pods per plant, NSPP - Number of seeds per plant, NSP - Number of seeds per pod, MSP - Mass of seeds per plant, M100 - Mass of hundred seeds, PH Plant height.

Table 8. Summary of variance analysis, adapted to partial diallels, between the Group 1 and 2 parents, and the heterosis deployment of its hybrids combinations.

\begin{tabular}{|c|c|c|c|c|c|c|c|}
\hline \multirow{2}{*}{ SV } & \multirow{2}{*}{ DF } & \multicolumn{6}{|c|}{ Mean square } \\
\hline & & NPP & NSPP & NSP & MSP (g) & M100 (g) & $\mathrm{PH}(\mathrm{cm})$ \\
\hline Treatments & 22 & $671.11^{* *}$ & $1268.49^{* *}$ & $0.214^{* *}$ & $19.00^{* *}$ & $4.03^{* *}$ & $137.32^{* *}$ \\
\hline $\mathrm{GCA}_{1}$ & 2 & $1132.60^{* *}$ & $2992.45^{* *}$ & $0.889^{* *}$ & $72.51^{* *}$ & $6.13^{* *}$ & $46.37^{* *}$ \\
\hline $\mathrm{G}_{1}$ vs $\mathrm{G}_{2}$ & 1 & $4304.77^{* *}$ & $3404.91^{* *}$ & $0.977^{* *}$ & $4.64^{\mathrm{ns}}$ & $54.81^{* *}$ & $1128.62^{* *}$ \\
\hline Heterosis $(\mathrm{H})(\mathrm{SCA})$ & 15 & $288.33^{* *}$ & $650.21^{* *}$ & $0.086^{* *}$ & $8.40^{* *}$ & $0.94^{* *}$ & $56.50^{* *}$ \\
\hline H Mean & 1 & $1911.58^{* *}$ & $3380.00^{* *}$ & $0.224^{* *}$ & $19.53^{* *}$ & $9.89^{* *}$ & $141.06^{* *}$ \\
\hline $\mathrm{H}$ varietal $\left(\mathrm{G}_{2}\right)$ & 4 & $36.28^{\mathrm{ns}}$ & $76.38^{\text {ns }}$ & $0.021^{\text {ns }}$ & $3.53^{\text {ns }}$ & $0.294^{*}$ & $62.58^{* *}$ \\
\hline H specific & 8 & $248.38^{* *}$ & $596.70^{* *}$ & $0.113^{* *}$ & $8.43^{* *}$ & $0.308^{*}$ & $48.76^{* *}$ \\
\hline Residue & 44 & 70.68 & 178.96 & 0.01 & 2.49 & 0.11 & 3.7 \\
\hline
\end{tabular}

SV-Source of variation, DF - Degrees of freedom, NPP - Number of pods per plant, NSPP - Number of seeds per plant, NSP - Number of seeds per pod, MSP - Mass of seeds per plant, M100 - Mass of hundred seeds, PH - Plant height, GCA 1 - General combining ability of Group 1, GCA - General combining ability of Group 2, SCA - Specific combining ability, $G_{1}$ - Group 1, G - Group 2, ${ }^{\text {,s }}$ - Non significant, ${ }^{* *} e^{*}$ - Significant at $p>0,01$ and $p>0,05$ by $F$ test, respectively. 
Table 9. Heterosis values $(\mathrm{h})$ in relation to the parents' means of groups 1 and 2 , resultant of the crossings between eight soybean cultivars, in a partial diallel.

\begin{tabular}{|c|c|c|c|c|c|c|c|c|c|c|c|c|}
\hline \multirow{2}{*}{ Crossings } & \multicolumn{2}{|l|}{ NPP } & \multicolumn{2}{|l|}{ NSPP } & \multicolumn{2}{|l|}{ NSP } & \multicolumn{2}{|c|}{ MSP (g) } & \multicolumn{2}{|c|}{ M100 (g) } & \multicolumn{2}{|c|}{$\mathrm{PH}(\mathrm{cm})$} \\
\hline & $\mathrm{h}$ & $\%$ & $\mathrm{~h}$ & $\%$ & $\mathrm{~h}$ & $\%$ & $\mathrm{~h}$ & $\%$ & $\mathrm{~h}$ & $\%$ & $\mathrm{~h}$ & $\%$ \\
\hline $\mathrm{G} 1 \times \mathrm{G} 4$ & 17.8 & 25.9 & 17.2 & 13.0 & -0.2 & -11.3 & 2.1 & 12.1 & -0.6 & -4.7 & -2.7 & -2.6 \\
\hline G1 x G5 & 4.7 & 6.3 & 10.5 & 8.3 & 0.0 & 0.0 & 1.1 & 6.2 & -0.5 & -3.8 & 5.3 & 5.1 \\
\hline $\mathrm{G} 1 \times \mathrm{G} 6$ & 24.3 & 30.0 & 5.8 & 4.1 & -0.4 & -19.6 & 0.1 & 0.4 & -1.1 & -7.4 & -1.0 & -1.0 \\
\hline G1x G7 & 13.2 & 16.6 & 22.8 & 17.2 & -0.1 & -0.9 & 1.5 & 8.0 & -1.5 & -9.8 & 2.5 & 2.5 \\
\hline $\mathrm{G} 1 \times \mathrm{G} 8$ & 6.5 & 9.7 & 9.3 & 7.9 & -0.1 & -2.8 & -0.4 & -2.4 & -1.6 & -10.6 & -0.5 & -0.5 \\
\hline $\mathrm{G} 2 \times \mathrm{G} 4$ & -5.2 & -8.1 & 1.3 & 0.9 & 0.1 & 6.5 & -0.2 & -1.2 & -0.4 & -3.4 & -0.2 & -0.2 \\
\hline $\mathrm{G} 2 \times \mathrm{G} 5$ & 15.7 & 22.7 & 30.3 & 0.1 & -0.1 & -6.3 & 2.4 & 13.1 & -1.0 & -7.6 & 4.2 & 4.0 \\
\hline $\mathrm{G} 2 \times \mathrm{G} 6$ & 5.3 & 7.0 & -0.3 & -0.2 & -0.3 & -13.0 & -0.7 & -3.6 & -0.6 & -4.6 & 7.5 & 7.7 \\
\hline G2x G7 & 1.8 & 2.5 & -3.0 & -2.1 & -0.3 & -13.4 & -0.8 & -4.2 & -0.4 & -2.4 & 13.0 & 13.4 \\
\hline G2x G8 & 12.5 & 20.1 & 2.8 & 2.2 & -0.4 & -19.1 & -1.1 & -6.2 & -1.1 & -8.4 & 1.3 & 1.4 \\
\hline $\mathrm{G} 3 \times \mathrm{G} 4$ & 23.7 & 33.0 & 35.2 & 24.1 & -0.2 & -7.3 & 4.1 & 21.3 & -0.5 & -3.3 & 5.8 & 5.6 \\
\hline $\mathrm{G} 3 \times \mathrm{G} 5$ & 5.5 & 7.2 & 13.5 & 9.6 & 0.0 & 1.8 & 0.6 & 2.9 & -0.9 & -6.4 & -2.2 & -2.0 \\
\hline $\mathrm{G} 3 \times \mathrm{G} 6$ & 16.5 & 19.7 & 46.5 & 29.6 & 0.1 & 6.1 & 5.6 & 25.7 & -0.7 & -4.8 & 6.2 & 6.2 \\
\hline G3x G7 & 17.7 & 21.5 & 15.5 & 10.6 & -0.2 & -11.7 & 1.6 & 7.8 & -0.5 & -3.4 & 9.7 & 9.7 \\
\hline G3x G8 & 9.3 & 13.3 & 17.7 & 13.4 & 0.0 & -0.9 & 1.2 & 6.2 & -0.9 & -6.0 & -3.0 & -3.1 \\
\hline
\end{tabular}

Table 10. Estimates of varieties effects $\left(v_{i}\right.$ and $\left.v_{j}\right)$ and varietal heterosis $\left(h_{i}\right.$ and $\left.h_{j}\right)$, between eight soybean parents, associated to groups 1 and 2 , respectively.

\begin{tabular}{|c|c|c|c|c|c|c|c|c|c|c|c|c|}
\hline \multirow[b]{2}{*}{ Group 1 (i) } & \multicolumn{2}{|l|}{ NPP } & \multirow{2}{*}{$\begin{array}{l}\text { NSPP } \\
v_{i}\end{array}$} & \multicolumn{2}{|c|}{ NSP } & \multicolumn{2}{|c|}{ MSP (g) } & \multicolumn{2}{|c|}{ M100 (g) } & \multicolumn{2}{|c|}{$\mathrm{PH}(\mathrm{cm})$} & \multirow[b]{2}{*}{$h_{i}$} \\
\hline & $v_{i}$ & $h_{i}$ & & $\mathrm{~h}_{\mathrm{i}}$ & $v_{i}$ & $h_{i}$ & $v_{i}$ & $\mathrm{~h}_{\mathrm{i}}$ & $v_{i}$ & $\mathrm{~h}_{\mathrm{i}}$ & $\mathrm{v}_{\mathrm{i}}$ & \\
\hline G1 & 1.44 & 2.01 & -16.11 & -1.87 & -0.36 & -0.01 & -1.46 & -0.25 & 0.97 & -0.25 & 0.22 & -2.33 \\
\hline $\mathrm{G} 2$ & -8.56 & -5.25 & 4.88 & -8.77 & 0.44 & -0.07 & -0.69 & -1.24 & -1.21 & 0.11 & -2.78 & 2.10 \\
\hline \multirow[t]{2}{*}{ G3 } & 7.11 & 3.24 & 11.22 & 10.65 & -0.08 & 0.07 & 2.15 & 1.47 & 0.23 & 0.14 & 2.55 & 0.23 \\
\hline & NPP & & NSPP & & NSP & & MSP & & $\mathrm{M} 100$ & & $\mathrm{PH}(\mathrm{cr}$ & \\
\hline Group $2(\mathrm{j})$ & $\mathrm{v}_{\mathrm{j}}$ & $\mathrm{h}_{\mathrm{j}}$ & $\mathrm{v}_{\mathrm{j}}$ & $h_{j}$ & $\mathrm{v}_{\mathrm{j}}$ & $h_{j}$ & $\mathrm{v}_{\mathrm{j}}$ & $h_{j}$ & $\mathrm{v}_{\mathrm{j}}$ & $h_{j}$ & $\mathrm{v}_{\mathrm{j}}$ & $h_{j}$ \\
\hline G4 & -10.40 & 0.82 & 3.13 & 2.87 & 0.26 & 0.05 & -1.42 & 0.87 & -1.14 & 0.31 & 4.00 & -2.06 \\
\hline G5 & -0.06 & -2.67 & -8.2 & 3.10 & -0.16 & 0.89 & -0.38 & 0.24 & 0.39 & -0.02 & 8.66 & -0.62 \\
\hline G6 & 13.93 & 4.10 & 25.80 & 2.32 & -0.07 & -0.05 & 2.88 & 0.49 & -0.18 & 0.03 & -2.66 & 1.15 \\
\hline G7 & 10.26 & -0.40 & 3.80 & -3.23 & -0.14 & -0.05 & 1.58 & -0.36 & 0.66 & 0.06 & -3.00 & 5.32 \\
\hline G8 & -13.73 & -1.84 & -24.53 & -5.06 & -0.07 & -0.04 & 2.68 & -1.25 & 0.26 & -0.36 & -7.00 & -3.79 \\
\hline
\end{tabular}

NPP - Number of pods per plant, NSPP - Number of seeds per plant, NSP - Number of seeds per pod, MSP - Mass of seeds per plant, M100 - Mass of hundred seeds, PH - Plant height.

for the characters related to yield described previously, and negative only for M100. Just as the yield components, this is a variable that suffers environmental influence, being necessary more attention for this parent in later studies.

It points out that the parent G4 obtained positive values of $\hat{g}_{i}$ for the characters NSPP and NSP and negative for NPP, reflecting in reduction of seeds production. It can be explained due to negative values for M100, contradicting Peluzio et al. (2005), that studied different soybean populations and the combinations between different characteristics, verifying that the selection of plants with higher NPP allows the indirect breeding for the characteristic grains yield.

These results indicates that the parents G3, G7 and G6 possess higher frequency of favorable alleles for the characteristics related to yield, remembering that the allelic frequencies of parents of a group are relative to the parents of other of other groups.

The estimates of SCA $\left(\hat{s}_{i j}\right)$ effects among parents of groups 1 and 2 can be visualized in Table 7. The effect of SCA is interpreted as the deviance of a hybrid in relation to what would be expected based on the GCA of its parents. Thus, lower absolute values of $\hat{s}_{i j}$ indicates that the $\mathrm{F}_{1}$ hybrids between the highlighted parents had an expected behavior based on its GCA, while higher absolute values of $\hat{s}_{i j}$ indicates that the behavior of a particular cross is relatively better or worse than the expected based on the GCA of its parents. The $\hat{s}_{i j}$ estimates evidenced the importance of genes that demonstrate non-additive effects (Cruz, Regazzi and Carneiro 2012).

According to the estimates of $\hat{s}_{i j}$ for the characters $\mathrm{G} 3 \times \mathrm{G} 6$, G2 x G5 and G3 x G4 with $\hat{s}_{i j}$ estimates of 3.76; 2.25 and

2.17, respectively. Cruz, Regazzi and Carneiro (2012) preconize that, for breeding endings, hybrids combining with elevated $\hat{s}_{i j}$ estimates and that involves at least a parent with high GCA are more desired. In this manner, the cross between $\mathrm{G} 3 \times \mathrm{G} 6$ and $\mathrm{G} 3 \times \mathrm{G} 4$ have a tendency to be promising, due to its elevated GCA presented by the parent G3.

For M100, all combinations had negative estimates of $\hat{s}_{i j}$, with exception to the crossings $\mathrm{G} 1 \times \mathrm{G} 5$ and $\mathrm{G} 2 \times \mathrm{G7}$, where the parent G7 presented GCA value more elevated (0.35), however, closer to the parent $\mathrm{G} 1(0.34)$. In this case, stands out the G2, which obtained negative estimate of GCA $(-0.54)$ while the parent $\mathrm{G} 5$ obtained positive estimate $(0.18)$,

indicating that the best combining for this characteristic was G1 x G5.

Considering simultaneously the characteristics NPP, NSPP and $\mathrm{PH}$, the populations resulting from the crossings between G3 x G4 and G2 x G5, are more promising for the obtaining of lines that group such phenotypes. 
Ramalho et al. (2012) affirm that, in the choice of segregating populations between those evaluated in the diallel, the decision must be focused in the GCA and SCA estimates. Still, according to the authors, the ideal is to identify a population in which the two parents present the higher $\hat{g}_{i}$ estimates and high SCA. When it occurs, the population has high mean, because the parents $\hat{g}_{i}$ are associated to frequency of favorable alleles and to high SCA indicates that the two parents complement themselves. In summary, the population would have large number of locus in heterozygosis, and, consequently, higher potential genetic variability, thus associating two fundamental criteria in the choice of population: high mean and higher genetic variance in $F \infty$.

\section{Heterosis estimates and effects}

For the studied variables, the heterosis dissolution is presented in Table 8. The deployment of the SCA effects in medium heterosis, varietal heterosis (attributed to many genotypes within each group) and specific heterosis justify only when the SCA presents significant effect (Silva, 2011). There was significant effect for mean heterosis of all variables. For the varietal heterosis of $\mathrm{G}_{1}$, all the variables presented significance, except for NPP. For the varietal heterosis of $G_{2}$, were only observed significant effects for the characters $\mathrm{M} 100$ and $\mathrm{PH}$. For the specific heterosis, the deployment had significance for all studied variables.

Based on the results it is possible to infer that, for the characters NSPP, NSP and MSP, only the parents of $G_{1}$ presented differentiated heterotic effects and, for M100 and $P H$, this conclusion is valid as much for $G_{1}$ and $G_{2}$.

The parents with higher effect of varietal heterosis are more divergent or its alleles present larger dominance deviances, compared to those of lower heterotic effect. Parents with higher diversity are required in crosses seeking to obtain transgressive segregation, indicating that the segregating populations will present greater potential to extract superior lineages (Cruz, Regazzi and Carneiro 2012). The significance of the specific heterosis effect for the characters NPP, NSPP, NSP, MSP and M100 evidences that the parents present non-allelic genes with epistatic interaction. It worth to point out the interactions types dominant $\mathrm{x}$ dominant, dominant $\mathrm{x}$ additive and additive $x$ dominant are not inheritable, being availed only by hybrids (Cruz, Regazzi and Carneiro 2012). The heterosis values in the crossings vary in magnitude and signal, as showed in the Table 9.

For the yield components, the cross G3 x G6 stands out since it obtained $29.6 \%$ of seeds plant ${ }^{-1}$ more than its parent, and a heterosis value of $6.6 \mathrm{~g} \mathrm{plant}^{-1}$, overtaking its parents means by $25.7 \%$. In the other hand, for NPP, the highlight for high heterosis was the cross $\mathrm{G} 3 \times \mathrm{G} 4$, being $33 \%$ superior to the parents, followed by the combination G1 x G6 with $30 \%$. For M100, all values were negative. The cross with greater impact for this variable was $\mathrm{G} 1 \times \mathrm{G} 8$, with reduction of $-1.6 \mathrm{~g}$ $(-10.6 \%)$, followed by the cross G1 x G7 with $-1.6 \mathrm{~g}(-9.8 \%)$.

However, it is opportune to point out that not always the hybrids with larger heterosis present superior means. It is because the superiority of a hybrid depends as much on the amount of locus in heterozygosis as the parents mean (Silva, 2011).

The estimate of varieties effects ( $v i$ and vj) of parents of each group is presented in Table 10. The effect per se of a parent in particular is an indicator of its superiority or inferiority in terms of favorable alleles frequency.

In the case of the variables NPP, NSPP and MSP, the parent $G 3$, from group $G_{1}$, and $G 6$, from group $G_{2}$, presented the larger effects (positive and high), as much for variety (vi and vj) as for varietal heterosis (hi and hj), indicating higher concentration of favorable alleles for these characteristics.

Considering the varietal heterosis effects (hi and hj), it was observed, for MSP, that the parents of group $G_{1}$ behaved different, pointing out to $G 3$, the only with positive value. Thus, at the $G_{2}$, stood out the parents $G 4, G 5$ and $G 6$. The rest of this group presented negative values for varietal heterosis. For NSPP, the highlight for $\mathrm{G}_{1}$ was the parent $\mathrm{G} 3$, with positive effect and above all. At the $G_{2}$, the parents $G 4$ and G5 were similar, being superior the parent G8, with negative value and inferior to the rest. For $\mathrm{PH}$, the parents G2 and G7 presented higher values of varietal heterosis associated with its respective groups.

\section{Materials and methods}

The experiment was carried out in the city of Iruna, located in the Department of Alto Paraná, Paraguay, at $330 \mathrm{~m}$ of altitude, 26010'S latitude and 55\%10' longitude. Eight soybean cultivars with known agronomic profile and recommended for cultivation in Paraguay, Argentina and Brazil were selected as parents (Table 1).

\section{Diallel procedure}

The parents were divided in two groups contrasting for the characteristics: mass of thousand seeds, maturity group, flowers color, lodging and growth aspect. The scheme of partial diallel followed the model 2 of Griffing (1956), where it were included the parents and the $F_{1}$ hybrids, totalizing $n(n+1) \cdot 0,5$ combinations (Table 1 ).

\section{The experiment}

With the aim of guarantee the use of pure genetic material in the hybridizations, it was proceeded the seeding, in greenhouse, of five seeds of each parent, in January 2012. During the development, the plants were collated with the expected phenotype through its morphologic characteristics. In the maturation, seeds originated from one plant were collected, and will origin the plants to be hybridized.

In May 2012, it was proceeded the seeding of the pure seeds, in greenhouse with automatic system of temperature control (variation of 18 to $30 \circ \mathrm{C}$ ). It were used plastic vases of 10 liters, containing soil mixture (70\%), organic matter (30\%) and $50 \mathrm{~g}$ of fertilizing NPK 04-30-10. The irrigation was realized by dripping system, during the whole cycle of the plants, keeping the soil in its field capacity. The supplemental artificial illumination was used to increase photoperiod in 4 hours per day in the vegetative stage and 2 hours per day at flowering, being interrupted at the end of hybridizations.

It was sown eight seeds of each parent in four vases and four sowing periods, inset on time (weekly), totaling 16 vases and 64 plants. This proceeding secured that the flowering periods were coincident, allowing the elongation of the hybridization procedure, according to Borém and Miranda (2009). 
By occasion of the maturity, it was proceeded the harvesting of pods from the hybridization (hybrid seeds or $F_{1}$ ). The seeds resulting from self-pollination were also collected for the sowing and evaluation of the respective parent at the field. In order to guarantee that the harvested seeds were not product of self-pollination it was used flower color as morphologic marker, which is a dominant character. Thus, the parents of group 1, with purple color, were used as pollen donors and crossed with the group 2 parents, with white flower color. As the flowering at field occurred, the $F_{1}$ plants were inspected, and proceeding the discard of the ones with white flowers.

The seeding took place in November 2012, manually, in prepared soil, free of clods and crop residues or straws that would interfere in the emergence. It was proceeded a broadcast fertilizing, a week previously to the seeding, with $200 \mathrm{~kg} \mathrm{ha}^{-1}$ of NPK $04-30-10$ fertilizing, according to soil analysis. The seeds did not receive any chemical or biologic treatment.

The experiment was placed in a seeds production field and the cultural management followed the recommendations for soybean production in the region, seeking to keep the plants with no disease and pests infestation. It was sown $15 F_{1}$ hybrids and its eight parents, using randomized blocks as experimental design, with three replicates. Due to the reduced number of seeds, the experimental units were composed of two meters length, spaced in $0,5 \mathrm{~m}$, with 10 seeds per meter. Seeking to avoid the border effect, it was sown an extra row between blocks. It was randomly choose ten plants to be evaluated, in the central part of each experimental unit.

The plants were harvested in its seeds were trashed and submitted to natural drying, until achieving a water content equal or inferior to $12 \%$. The following variables were measured: a) number of pods per plant (NPP); b) number of seeds per plant (NSPP); c) number of seeds per pod (NSP): total number of seeds divided by the total number of pods per plant; d) mass of seeds per plant (MSP); e) mass of hundred seeds (M100) and f) plant height (PH) in R8. The characteristics NPP, NSPP, NSP, M100 and AP were correlated with MSP to verify the possibility of use in the selection of higher productive plants.

\section{Statistical procedures}

The data was submitted to variance analysis. The parents and $F_{1}$ plants means were analyzed according to the partial diallel model proposed by Geraldi and Miranda Filho (1988), and Miranda Filho and Geraldi (1984) adapted from the models proposed by Griffing (1956), and Gardner and Eberhart (1966), respectively. The statistical analysis were realized in the software GENES (Cruz, 2006).

\section{Conclusions}

There was predominance of additive genic effects for the characteristics number of seeds per pods, number of seeds per plant and plants height. For number of pods per plant, seeds per plant and mass of hundred seeds there was predominance of dominance effects, expressed by the superiority of SCA in the determination of these characters. The parents G3 and G7 were the ones that contributed the most to the increase in yield, in function of GCA for the characters number of pods per plant, seeds per plant, plant yield and mass of hundred seeds.

The hybrids resultants from the crossings between $\mathrm{G} 3 \times \mathrm{G} 5$ and G3 x G4 are more promising for the characteristics related to yield, because they present elevated heterosis effect and high SCA associated to increase GCA presented by the parent G3.

\section{Acknowledgements}

To the National Council for Scientific and Technological Development (CNPq) and to Coordination for the Improvement of Higher Level -or Education- Personnel (CAPES), for the scholarships granted.

\section{References}

Baldissera JNC, Valentini G, Coan MMD, Almeida CB, Guidolin AF, Coimbra JLM (2012) Capacidade combinatória e efeito recíproco em características agronômicas do feijão. Semina: Ciên Agrárias. 33(2):471-480.

Borém A, Miranda GV (2009) Melhoramento de plantas. 5a ed. Viçosa: UFV. 529 p.

Carvalho IR, Nardino M, Demari G, Szareski VJ, Aumonde TZ, Pedo T, Monteiro MA, Pelegrin AJ, Olivoto T, Ferrari M, Meira, D, Souza VQ (2016) Biometry and Genetic Breeding of Dual-purpose Wheat. Inter J of Current Res. 8:343934545.

Carvalho IR, Nardino M, Souza VQ (2017) Melhoramento e Cultivo da Soja. 1. ed. Porto Alegre: Cidadela. v. 100. 366p .

Carvalho IR, Szareski VJ, Mambrin RB, Ferrari M, Pelegrin A, Corazza T Peter, M, Silveira, DC, Conte, GG, Barbosa, MH, Souza, VQ (2018) Biometric Models and Maize Genetic Breeding. Aust J of Crop Sci. 12:1796-1805.

Cruz CD. (2006) Programa Genes - Aplicativo computacional em genética e estatística. Availableat: www.ufv.br/dbg/genes/genes.htm.

Cruz CD, Regazzi AJ, Carneiro PCS (2012) Modelos biométricos aplicados ao melhoramento genético. 4ํㅡㄹ $\mathrm{ed}$. Viçosa: UFV. 514 p.

Cruz MFA, Souza GA, Rodrigues FA, Sediyama CS, Barros EG (2011) Reação de genótipos de soja à infecção natural por ferrugem asiática. Pesq Agropec Bras. 46:215-218.

Daronch DJ, Peluzio JM, Afférri FS, Nascimento MO (2014) Capacidade combinatória de cultivares de soja em F2, sob condições de cerrado tocantinense. Biosc J. 30(5): 688695.

Gardner CO, Eberhart SA (1966) Analysis and interpretation of variety cross diallel and related populations. Biometrics. 22(3): 439-452.

Geraldi IO, Miranda-filho JB (1988) Adapted models for the analysis of combining ability of varieties in partial diallel crosses. Braz J of Gen. 11(1):419-430.

Griffing B (1956) Concept of general and specific combining ability in relation to diallel crossing systems. Aust J of Biol Sci. 9(4):463-493.

Lorencetti C, Carvalho FIF, Benin G, Marchioro VS, Oliveira AC, Silva JAG, Hartwig, I, Schmidt, DAM, Valério, IP (2005) Capacidade combinatória e heterose em cruzamento dialélico de aveia (Avena sativa L.). Rev Bras de Agroc. 11(2): 143-148. 
Mebrahtu T, Devine TE (2009) Diallel analysis of sugar composition of 10 vegetable soybean lines. Plant Breed. 128: 249-252.

Miranda-Filho JB, Geraldi 10 (1984) An adapted model for the analysis of partial diallel cross. Braz J of Gen.7:677-688.

Morceli Junior AA, Di Mauro AO, Unêda-Trevisoli SH, Muniz FRS, Costa MM, Morceli TGS (2008) Análise genética em cruzamentos de soja com fonte de resistência ao nematóide de cisto. Rev Ceres. 55(3):153-159.

Nassar MAA (2013) Heterosis and combining ability for yield and its components in some crosses of soybean. Aust $\mathrm{J}$ of Basic and Applied Sci. 7(1): 566-572.

Polizel AC, Juliatti FC, Hamawaki OT, Hamawaki RL, Guimarães SL (2013) Adaptabilidade e estabilidade fenotípica de genótipos de soja no estado de Mato Grosso. Biosc J. 29(4):910-920.
Peluzio JM (2005) Correlações entre caracteres de soja, em Gurupi, Tocantins. Rev Ceres. 52(303):779-786.

Ramalho MAP, Abreu AFB, Santos JB, Nunes JAR (2012) Aplicações da genética quantitativa no melhoramento de espécies autógamas. 1a ed. Lavras: UFLA, 522 p.

Silva VMP (2011) Melhoramento genético do porte do feijoeiro. 2011. 73 f. PhD Theses - Universidade Federal de Viçosa, Viçosa.

Valério IP, Carvalho FIF, Oliveira AC,Velci QS, Benin G, Schmidt AM, Ribeiro, G. R.; Nornberg, R.; Luch, H (2009) Combining ability of wheat genotypes in two models of diallel analyses. Crop Breed and Appl Biotec. 9: 100-107.

Zorzetto MM, Costa Motta F, Morais LK, Kiihl TAM, Silva RM (2008) Análise dialélica da capacidade combinatória em soja. Rev Bioc. 14(2): 105-109. 